\title{
Traffic Management Heuristics for Bidirectional Segments on Double-Track Railway Lines*
}

\author{
Norman Weik, Stephan Zieger, and Nils Nießen \\ Institute of Transport Science, RWTH Aachen University, 52062 Aachen, Germany \\ weik@via.rwth-aachen.de, \\ WWW home page: http://www.via.rwth-aachen.de
}

\begin{abstract}
In this paper traffic management strategies for disruptioncaused inaccessibility of one track on double-track railway lines are analyzed. Building on previous results for polling systems with $k_{i}$-limited service discipline and non-zero switchover times heuristic rules for optimally switching the orientation of the remaining track are investigated. QoS-standards enforcing delay constraints for both orientations as well as asymmetric traffic load, e.g. resulting from differing block length in both directions, are accounted for. For a system without train priorities analytical approximations as well as simulation results for the optimal switching parameter are compared. The analysis is extended to priority systems in a simulation approach. The performance of the heuristic is tested in a case study by comparing it to the optimal solution obtained by solving the train scheduling problem for the bidirectional line segment.
\end{abstract}

Keywords: transportation, railway, disruption, polling, control, delay, priorities, constrained optimization

\section{Introduction}

Temporary closures of one track on double track railway lines pose a severe, yet relatively frequent event on Europe's dense and heavily loaded railway networks. They may arise from train or infrastructure malfunctions or maintenance requirements. Traffic management on the remaining bidirectional track, including determining the admissible traffic load and the train sequence through the bottleneck is a demanding task for dispatchers.

The present work aims to investigate heuristic traffic management strategies for this situation based on a polling system perspective. A polling system corresponds to a queuing system where a single server serves multiple queues. The order in which the server visits (polls) the different queues is given by the polling table, whereas the time spent at a given queue is determined by the polling policy. When changing from one queue to another a switchover time, where the server does not perform service may be inserted. In addition, the server may need to set up upon arrival at a queue.

In transportation, polling systems have been widely used to analyze waiting times at traffic signals [3], where setup and switchover times are small. The railway case, however, is more closely related to construction zones or underground

\footnotetext{
* This is a pre-copyedited version of a contribution published in Kliewer N., Ehmke J., Borndörfer R. (eds) Operations Research Proceedings 2017, published by Springer, Cham. The definitive authenticated version is available online via https://doi.org/ 10.1007/978-3-319-89920-6_97
} 
transport systems, for which approximations of the mean waiting times have been derived in [6] assuming fix service times and switching intervals.

We subsequently take a more general perspective based on polling models with arbitrary switchover and service times and $k_{i}$-limited service policy. This class of models does not satisfy the branching property, rendering the solution difficult. Our work is based on previous work by Borst et al. [1], where the authors discuss approximation approaches for finding the set of control parameters $k_{1}, \ldots, k_{N}$ that minimizes the expected waiting time. More recently, van Vuuren et al. [7] have presented an iterative approach to determine the queue length distributions for $k_{i}$-limited policy. The $N$-queue polling system is modeled by single queues with server vacations, where vacation times are matched to conditional interarrival times. Vacation, service and arrival intervals are approximated by phase-type distributions and the resulting QBD model is solved using psa [7].

The present work bridges the gap between theory and railway applications. While queueing models have found widespread applications in railway capacity analysis, the use of polling models is new in this context. The contribution of the present paper includes three aspects: First, we compare analytical results based on the Fuhrmann-Wang approximation [4] with simulation results for realistic train programs. In a second step we discuss how priorities, which are typical for railways, affect the results obtained in the previous case. Finally, we analyze the performance of the heuristic, where line orientation is changed according to the optimal control parameters in the polling model.

\section{Model}

\subsection{Polling Model}

We subsequently model the bidirectional line segment as a polling system with a single server and two queues. Service times correspond to headway times between successive trains. The two queues are served according to a $k_{i}$-limited policy, i.e. the server serves at most $k_{i}$ trains at a queue and switches to the other queue if the current queue runs empty. The vastly different train separation times between trains of same and opposing directions are modeled using switchover times. Setup times upon arrival at a new queue are not considered. Arrivals are assumed Markovian - modeling the fact that information is scarce in case of heavily perturbed operations. For service and switchover times general independent distributions are admitted.

Notation: For queue $i$, we denote traffic load by $\rho_{i}$, the means of service and switchover times by $\beta_{i}$ and $s_{i}$ and arrival rates by $\lambda_{i}$. The second moments of service and switchover times are denoted by $\beta_{i}^{(2)}$ and $s_{i}^{(2)}$, respectively. The total switchover time and traffic load in the system is given by $s=\sum s_{i}$ and $\rho=\sum \rho_{i}$.

Stability: For $k_{i}$-limited polling models a necessary and sufficient stability criterion reads $\rho+s \cdot \max _{i} \frac{\rho_{i}}{\beta_{i} k_{i}}<1$ [3]. This already enforces a lower bound on the control parameter $k_{i}: k_{i} \geq s \cdot \frac{\rho_{i}}{\beta_{i}(1-\rho)} \forall i$. 


\section{$2.2 \quad$ Waiting Times}

In [1], Borst et al. compare various approximation formulae for the expected waiting times in $k_{i}$-limited polling models. As $k$-limited systems do not satisfy the branching theorem, no exact formulae for the waiting times are known. We subsequently adopt Approximation (4) in [1], which has been shown to perform best [1]. It has been derived by Fuhrmann and Wang [4] from the pseudo-conservation law for the work as an upper bound on waiting times in $k$-limited polling systems. While it does not yield a closed formula for the optimal set $k$, the latter can easily be obtained numerically for a small number of queues.

\section{Problem Definition}

The traffic management goals are to minimize overall delays while limiting the maximal waiting time for passengers. The quality of service for passengers is ensured by limiting the interarrival time to a given queue, s.t. the time interval between two succeeding trains in the same direction is bounded. In the following we discuss two problems arising in this context.

\subsection{Problem 1}

Determine the set of control parameters $k_{i}$ that minimizes the expectation of the overall waiting times under the constraint that the interarrival time in case of overload is bounded by some $C>0$ on average:

$$
k_{i} \cdot \beta_{i}+s \leq C .
$$

This problem is a variant of the constrained optimization problem in [1].

\subsection{Problem 2}

Determine the fairest set of control parameters $k_{i}$, i.e. choose $k$ such that the maximum expected interarrival time $\max _{i} E\left[I_{i}\right]$ is minimized, subject to waiting time constraints $E\left[W_{i}\right]<K$ for all queues $i$ and some $K>0$.

\subsection{Input Data}

The input data is derived from typical train operating concepts on mixed service railway lines in Europe. For the bidirectional segment different lengths between 3 and $15 \mathrm{~km}$ and trains with velocities between 80 and $230 \mathrm{~km} / \mathrm{h}$ are considered, which gives rise to different service and switchover times in the model.

For the service times of trains traveling in the same direction two profoundly different scenarios can be distinguished. In Scenario 1, the remaining track is equipped with signaling, and hence multiple block segments, in both directions. In this case, the polling system can be assumed to be symmetric as minimum headways in both direction are approximately equivalent. Scenario 2 corresponds to vastly different service times at queue 1 and 2, which occurs if the remaining track is not equipped with signaling in the irregular direction, such that it can only be used one train at a time in this direction. 


\section{Results}

We subsequently present numerical results for the symmetric case (Scenario 1) and the asymmetric case (Scenario 2). In Section 4.1 the results based on the Fuhrmann-Wang Approximation are compared to simulation results of pollingbased train operations. If both queues run empty in the simulation the server continues at the queue with the next possible service.

In Section 4.2 the effects of train priorities are discussed and in Section 4.3 the performance of the polling heuristic with optimal $k$ in Problem 1 is compared to the solution for the train scheduling problem.

\subsection{Fuhrmann-Wang Approximation for Waiting Times}

Problem 1: The solution of Problem 1 in the symmetric scenario can be given analytically. As both the simulated and the approximated waiting time are decreasing in the control parameter $k[1]$, the solution corresponds to $k=\left\lfloor\frac{C-s}{\beta}\right\rfloor$.

For Scenario 2 we consider input parameters resulting from different lengths of the single-track segment as well as different train velocities. An excerpt of the results with $C=30 \mathrm{~min}$ is presented in Table 1 .

Table 1. Excerpt of results for asymmetric load in Scenario 2.

\begin{tabular}{c|c|c|c}
$\rho=0.58, s=1.19$, & $\rho=0.68, s=3.78$, & $\rho=0.73, s=5.99$, \\
$\beta=(2.5,3.69)$, & $\beta=(3.01,6.79)$, & $\beta=(3.46,9.45)$, \\
& $s^{(2)}=1.54$, & $s^{(2)}=16.29$, & $s^{(2)}=40.41$, \\
& $\beta^{(2)}=(6.25,13.78)$ & $\beta^{(2)}=(10.16,48.20)$ & $\beta^{(2)}=(14.90,94.93)$ \\
\hline$k_{\text {simu }}$ & $(11,7)$ & $(8,3)$ & $(6,2)$ \\
$k_{\text {approx }}$ & $(11,7)$ & $(8,3)$ & $(6,2)$ \\
\hline
\end{tabular}

Our results suggest that for the asymmetric scenario with different $\beta_{i}$ the optimal strategy corresponds to taking the highest admissible $k_{i}$ that satisfies the constraints. In [1], it was shown for the unconstrained problem that $\sum_{j} \rho_{j} E\left[W_{j}\right]$ is non-increasing in $k_{i}$. The experiments we conducted seem to indicate that this strategy is also close to minimizing the constrained waiting times.

Problem 2: For Problem 2, the results for $K=30 \mathrm{~min}$, which is a typical train frequency in railway operations, are depicted in Table 2. For both scenarios results with a homogeneous and a heterogeneous traffic mix are shown. The first one is based on a segment of $8 \mathrm{~km}$ and train speed differences of max. 60 $\mathrm{km} / \mathrm{h}$, the second one to a $15 \mathrm{~km}$ segment and very distinct speed differences of up to $130 \mathrm{~km} / \mathrm{h}$. The variation coefficients of service and switchover times are $\left(v_{B}=0.2, v_{S}=0.14\right)$ and $\left(v_{B}=0.53, v_{S}=0.25\right)$.

It seems that while the results of the Fuhrmann-Wang Approximation [4] are reasonably good for intermediate and low traffic load the approximated results 
Table 2. Optimal set of parameters in symmetric and asymmetric scenario. Comparison of simulation and approximate results. In Scenario 2, the scheduled traffic load $\rho$ increases to $\rho_{\text {act }}$ due to the disruption-caused change of service times.

\begin{tabular}{|c|c|c|c|c|c|c|c|c|c|c|c|c|}
\hline & & $\rho$ & 0.30 & 0.35 & 0.40 & 0.45 & 0.50 & 0.55 & 0.60 & 0.65 & 0.70 & 0.75 \\
\hline \multirow{4}{*}{ 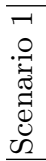 } & \multirow{2}{*}{ small var. } & $k_{\text {simu }}$ & 1 & 1 & 2 & 2 & 2 & 3 & 3 & 4 & 5 & 7 \\
\hline & & $k_{\text {approx }}$ & 1 & 2 & 2 & 2 & 3 & 3 & 4 & 5 & 6 & 9 \\
\hline & \multirow{2}{*}{ high var. } & $k_{\text {simu }}$ & 1 & 2 & 2 & 2 & 3 & 3 & 4 & 5 & 6 & 9 \\
\hline & & $k_{\text {approx }}$ & 2 & 2 & 2 & 3 & 3 & 4 & 5 & 7 & 9 & 15 \\
\hline \multirow{6}{*}{ 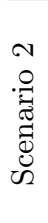 } & \multirow{3}{*}{ small var. } & $\rho_{a c t}$ & 0.45 & 0.52 & 0.58 & 0.64 & 0.70 & 0.76 & \multirow{3}{*}{$\begin{array}{c}0.81 \\
(4,5) \\
(5,6) \\
\end{array}$} & \multirow{6}{*}{\multicolumn{3}{|c|}{ infeasible }} \\
\hline & & $k_{\text {simu }}$ & $(1,1)$ & $(1,1)$ & $(1,1)$ & $(1,2)$ & $(2,2)$ & $(3,3)$ & & & & \\
\hline & & $k_{\text {approx }}$ & $(1,1)$ & $(1,1)$ & $(1,1)$ & $(2,2)$ & $(2,2)$ & $(3,4)$ & & & & \\
\hline & \multirow{3}{*}{ high var. } & $\rho_{a c}$ & 0.48 & 0.55 & 0.61 & 0.68 & 0.74 & 0.79 & & & & \\
\hline & & $k_{\text {simu }}$ & $(1,1)$ & $(1,1)$ & $(1,2)$ & $(2,2)$ & $(3,4)$ & $(4,6)$ & & & & \\
\hline & & $k_{\text {approx }}$ & $(1,1)$ & $(1,2)$ & $(2,2)$ & $(2,3)$ & $(4,4)$ & $(6,8)$ & & & & \\
\hline
\end{tabular}

deviate for large $\rho$. This suggests that the bound obtained from the approximation might not be as tight in the regime of high load and small switchover and service times variation. For this regime, only limited results have been presented in [1] as the analysis is numerically costly due to the large state space.

\subsection{On the Effects of Train Priorities on the Control Strategy}

In practice, railway dispatchers often employ a priority strategy, where faster passenger trains are given precedence over slower trains. It is unclear, how this affects the waiting times of trains. It is assumed that

- amongst waiting trains high-priority train are given preference over lowpriority trains of the same direction.

- high-priority trains waiting at a queue which currently does not receive service do not enforce early switchovers from the other queue.

Our simulation results for this type of system show that waiting times tend to decrease if priorities are considered. This can probably be explained by the grouping of trains with similar velocities leading to globally smaller service times. The effect is more pronounced the higher the system load and variation of service times. For typical mixed-service operations with $20 \%$ priority trains, $\rho \leq 0.6$ and $E\left[W_{i}\right] \leq 30$ min the size of the effect is found to be in the range of $2-9 \%$.

\subsection{Comparison Polling Heuristic and Scheduling Solution}

The performance of the polling heuristic with optimal $k$ in Problem 1 is analyzed in a case study by comparing the results to the optimal scheduling solution for a line segment. The time frame includes 17 trains in each direction and arrival intervals are rescaled to account for different $\rho$. Average service and switchover times are $3 \mathrm{~min}$ and $8 \mathrm{~min}$, respectively, and $k_{i}$ is taken to be 5 for all queues. For the optimization Castillo et al. [2] has been adopted and solved with Gurobi [5]. 
The objective is to minimize the sum of differences of actual starting time and release time of trains. Train priorities are modeled by increasing the weight in the objective function by $10 \%$. In the polling simulation both the performance for the schedule time frame as well as the long turn average (lt) obtained by periodically repeating the schedule are calculated.

Table 3. Comparison avg. waiting times polling-heuristic and optimization approach.

\begin{tabular}{c|ccccc}
$\rho$ & 0.53 & 0.56 & 0.58 & 0.61 & 0.64 \\
\hline$T_{W, \text { sched }}$ & 7.81 & 8.59 & 9.40 & 10.13 & 10.84 \\
$T_{W, \text { heuristic }}$ & 10.22 & 9.24 & 11.07 & 13.38 & 12.86 \\
$T_{W, \text { heuristic (lt) }}$ & 11.99 & 12.51 & 13.06 & 13.78 & 15.13
\end{tabular}

For the same schedule structure and different $\rho$, Table 3 shows that the polling heuristic performs roughly $10-30 \%$ worse than the optimal solution. However, for the time frame of ca. 3 hours the heuristic results exhibit huge variation as they depend on switching decisions. Monotonicity of waiting times is restored in longer time frames as $T_{W \text {,heuristic }(l t)}$ shows. Still, even for the relatively short schedule, the optimization for $\rho>0.6$ takes more than 12 hours, whereas the polling simulation including long turn average can be calculated within seconds.

\section{Conclusion}

We have adapted k-limited polling models for analysis and control of singletrack line segments of railway lines. The polling-based heuristic provides a fast and easy way to determine maximum admissible traffic load and efficient traffic management. For the case study considered, the results imply that the quality of the solution is roughly $10-30 \%$ above the optimal solution, hence it can only be considered a rough estimate for the sequencing problem if information is scarce.

Acknowledgement: This work was supported by DFG grant 283085490 .

\section{References}

1. Borst, S.C., Boxma, O.J., Levy, H., "The Use of Service Limits for Efficient Operation of Multistation Single-Medium Communication Systems". IEEE/ACM Transactions on Networking 3(5), pp. 602-612, 1995.

2. Castillo, Enrique, et al. "Timetabling optimization of a single railway track line with sensitivity analysis." TOP 17(2), pp. 256-287, 2009.

3. Frigui, I., "Analysis of a time-limited polling system with Markovian Arrival Process and phase type service". PhD thesis, University of Manitoba, Winnipeg, 1997.

4. Fuhrmann, S.W., Wang, Y.T.: Analysis of cyclic service systems with limited service: bounds and approximations. Performance Evaluation 9(1), pp. 35-54, 1988.

5. Optimization, Gurobi. Inc., "Gurobi optimizer reference manual", 2014.

6. Van der Heijden, M., van Harten, A., Ebben, M., "Waiting times at periodically switched one-way traffic lanes", Probab. Eng. Inform. Sc. 15(4), pp. 495-517, 2001.

7. Van Vuuren, M., Winands, E.M.M, "Iterative approximation of k-limited polling systems", Queueing Systems 55(3), pp. 161-178, 2007. 